\title{
Effect of strontium inclusion on the bioactivity of phosphate-based glasses
}

\author{
J. K. Christie ${ }^{1,3, *}$ (1) and N. H. de Leeuw $w^{2,3}$ \\ ${ }^{1}$ Department of Materials, Loughborough University, Loughborough LE11 3TU, UK \\ ${ }^{2}$ School of Chemistry, Cardiff University, Main Building, Park Place, Cardiff CF10 3AT, UK \\ ${ }^{3}$ Department of Chemistry, University College London, 20 Gordon Street, London WC1H OAJ, UK
}

Received: 27 October 2016

Accepted: 27 April 2017

Published online:

11 May 2017

(C) The Author(s) 2017. This article is an open access publication

\begin{abstract}
We have conducted first-principles and classical molecular dynamics simulations of various compositions of strontium-containing phosphate glasses, to understand how strontium incorporation will change the glasses' activity when implanted into the body (bioactivity). To perform the classical simulations, we have developed a new interatomic potential, which takes account of the polarizability of the oxygen ions. The Sr-O bond length is $\sim 2.44-2.49 \AA$, and the coordination number is 7.5-7.8. The $\mathrm{Q}^{n}$ distribution and network connectivity were roughly constant for these compositions. Sr bonds to a similar number of phosphate chains as Ca does; based on our previous work (Christie et al. in J Phys Chem B 117:10652, 2013), this implies that $\mathrm{SrO} \leftrightarrow \mathrm{CaO}$ substitution will barely change the dissolution rate of these glasses and that the bioactivity will remain essentially constant. Strontium could therefore be incorporated into phosphate glass for biomedical applications.
\end{abstract}

\section{Introduction}

Strontium is of profound importance in biomedical science [1], particularly because of its effect on bone growth. Strontium is naturally occurring in the human body, although at very low quantities, where it acts as a "bone seeker" like calcium, to the point that $\sim 99 \%$ of the body's strontium is found in bone. Strontium is known to act synergistically with calcium to promote osteoblastic bone formation as well as inhibiting osteoclastic bone resorption [2]. As a result, strontium ranelate is an approved treatment for osteoporosis in postmenopausal women [3-6], who have an increased prevalence of the disease, whereas strontium malonate is currently undergoing clinical trials. Strontium intake also leads to greater deposition of calcium in bone [1]. Other medical uses of strontium include exploiting its bactericidal nature for injectable bone cement [7] and as an adjunct to radiotherapy [8].

In recent years, there has been substantial interest in incorporating strontium into other therapeutic materials which are implanted into the body. The dissolution products of many such materials are also known to promote new bone growth $[9,10]$. Experimental [11, 12] and computational [13, 14] studies on

Address correspondence to E-mail: j.k.christie@lboro.ac.uk 
strontium-containing bioglass have shown that the effect of strontium inclusion on the atomic structure is rather small, even for quite large $(\leq 25 \%)$ concentrations of $\mathrm{SrO}$ [15]. However, the inclusion of $\mathrm{Sr}$ decreases bioactivity by decreasing the apatite-forming ability and dissolution rate [12] of bioglass.

Phosphate-based bioactive glasses (PBG) are also of considerable medical use, particularly because their dissolution rate is much faster than that of silicatebased glasses, and this rate can be tuned over several orders of magnitude by changes in the glass composition $[9,16]$. This allows for the possibility of delivery of therapeutic ions to a localised site in the body at an appropriate rate, for the control of bone growth [10] among other treatments. It is natural therefore to ask whether Sr's osteogenic properties could be combined with the favourable dissolution behaviour of PBG. To do this, however, we need to know the effect of strontium inclusion on the structure and degradation properties of PBG.

When $\mathrm{Sr}^{2+}$ was incorporated into $\left(\mathrm{P}_{2} \mathrm{O}_{5}\right)_{50^{-}}$ $\left(\mathrm{Na}_{2} \mathrm{O}\right)_{20}-(\mathrm{CaO})_{30}$ glasses, a disproportionation of $Q^{2}$ phosphorous atoms was seen, decreasing their prevalence and increasing the amount of $Q^{1}$ and $Q^{3}$ species [17]. Unexpectedly, the glass degradation rate increased with the addition of $1 \mathrm{~mol} \% \mathrm{SrO}$ (in place of $\mathrm{Na}_{2} \mathrm{O}$ ) and then decreased with the addition of 3-5 $\mathrm{mol} \% \mathrm{SrO}$, but it seems likely that this is due to varying phosphate contents (Table 4, Ref. [17]) in the prepared Sr-containing glasses, rather than any effect of the $\mathrm{Sr}$ itself. However, $\mathrm{SrO}$ substitution for $\mathrm{CaO}$ in Ti-containing ternary metaphosphate glasses seemed to show similar properties, with an increase by an order of magnitude in the degradation rate going from Sr-free to $1 \mathrm{~mol} \% \mathrm{SrO}$, and then a slight decrease for $\mathrm{SrO}$ contents up to $5 \mathrm{~mol} \%$, with similar trends exhibited for ion release [18, 19]. The Sr-containing glasses were also found to be biocompatible, with excellent cell attachment and growth properties.

It is clear that the precise effect of strontium inclusion on phosphate-based bioactive glasses is not fully understood. If Sr-containing PBGs are to be used for the controlled release of therapeutic ions, then the connections between the glass composition, atomic structure and macroscopic properties such as degradation rate and cell adhesion must be understood, and computer simulation offers a route to achieve this goal. Molecular dynamics (MD) simulations provide the atomic structure of the glass, as well as enabling computation of large-scale materials properties. We and others have previously used MD simulations to elucidate the connection between atomic structure and dissolution behaviour of a variety of silicate [20-27] and phosphate glasses [28-30] intended for implantation, including strontium-containing silicate glass $[13,14]$. However, there have been no simulations of $\mathrm{Sr}$ in phosphate glass.

In MD, the positions of the atoms evolve under Newton's laws of motion. First-principles MD relies on a quantum-mechanical description of the interatomic forces, provided here through density functional theory (DFT). Although very accurate, the computational expense of such simulations is very high, and they are therefore limited to small models (a few hundreds of atoms) and short timescales. In classical MD, the interatomic forces are approximated by an empirical potential which is easier to compute, permitting the simulation of much larger models for longer timescales, but at the cost of introducing approximations and losing information on the electronic structure. It is therefore vital to derive a potential that is as accurate as possible. Previous simulations of Sr-containing silicate glasses [13, 14] have used a rigid-ion interatomic potential, in which the ions have partial charges and the polarizability of the ions is not included. Although this can often give satisfactory agreement with experimental data [22], it has been shown [31] that using a potential which incorporates the polarizability of the oxygen atoms, typically using the shell model, provides a more accurate representation of the medium-range structure of glasses, particularly the network connectivity and $Q^{n}$ distribution. The network connectivity, $Q^{n}$ distribution [21] and other medium-range structural motifs [28] are known to affect the bioactivity substantially. It is therefore essential that in any simulation, they are described correctly. We have therefore developed a polarizable potential for strontium, which is compatible with other potentials [32], and will offer an accurate description of the relevant structures and properties.

We have shown [28] that for polyphosphate $\left(<50 \mathrm{~mol} \% \quad \mathrm{P}_{2} \mathrm{O}_{5}\right)$ glasses, in which the $\mathrm{PO}_{4}$ phosphate tetrahedra form finite-length unbranched chains, the dissolution rate is largely controlled by the interactions between these phosphate chains and the network-modifying cations (e.g., $\mathrm{Na}, \mathrm{Ca}$ ) to which they are bound. $\mathrm{Ca}$ binds to more chains than $\mathrm{Na}$, and this strongly contributes to the known decrease in degradation rate [9] when $\mathrm{Ca}$ is substituted for $\mathrm{Na}$, 
by strengthening the glass network. Since these compositions are commonly used [9], a full description of the effect of strontium inclusion on the dissolution of these glasses must therefore include its bonding to these chains.

In this work, we use classical and first-principles molecular dynamics to simulate several compositions of Sr-containing phosphate-based glasses. Following a description of the construction and validation of the new strontium potential, we describe the short-range structural environment around strontium, in terms of bond lengths, coordination environment and bond angles, before moving on to discuss the effect of strontium inclusion on the $Q^{n}$ distribution and network connectivity. We conclude with a discussion of strontium's effect on the suitability of the glass for biomedical implantation.

\section{Development of a strontium interatomic potential}

We sought to develop an accurate interatomic potential to take account of the inclusion of strontium into the glass structure. In our classical MD simulations, the interatomic interactions are described by the Born model of solids using full-charge pair potentials. In addition to the electrostatic interactions between ions, the short-range interactions are modelled by the Buckingham potential $V_{i j}(r)=A_{i j}$ $\exp \left(-r / \rho_{i j}\right)-C_{i j} / r^{6}$, where $r$ is the interatomic distance between atoms of species $i$ and $j$ and $A_{i j}, \rho_{i j}$ and $C_{i j}$ are the parameters of the model. Our criteria were to develop a potential compatible with previous polarizable potentials developed for both phosphate [32] and silicate [33] glasses, so that it could be used to model the strontium environment in both types of glasses. (This work studies only phosphate glasses; $\mathrm{Sr}$ in silicate glasses will be the subject of future work.)

The GULP code [34] was used to fit the Sr-Os Buckingham potential to reproduce the crystal structures of $\mathrm{SrO}$ [35], $\mathrm{SrSiO}_{3}$ [36], $\mathrm{Sr}_{2} \mathrm{SiO}_{4}$ [37] and $\mathrm{Sr}_{3}\left(\mathrm{PO}_{4}\right)_{2}$ [38], i.e., including oxide, silicate and phosphate environments. The other potential interactions were held constant to the values (Table 1) which have previously been used to model a wide range of silicate and phosphate glasses [32, 39]. The final potential parameters are listed in Table 1, and the lattice parameters of the crystalline structures are given in Table 2.
Table 1 The interatomic potential parameters used in this work

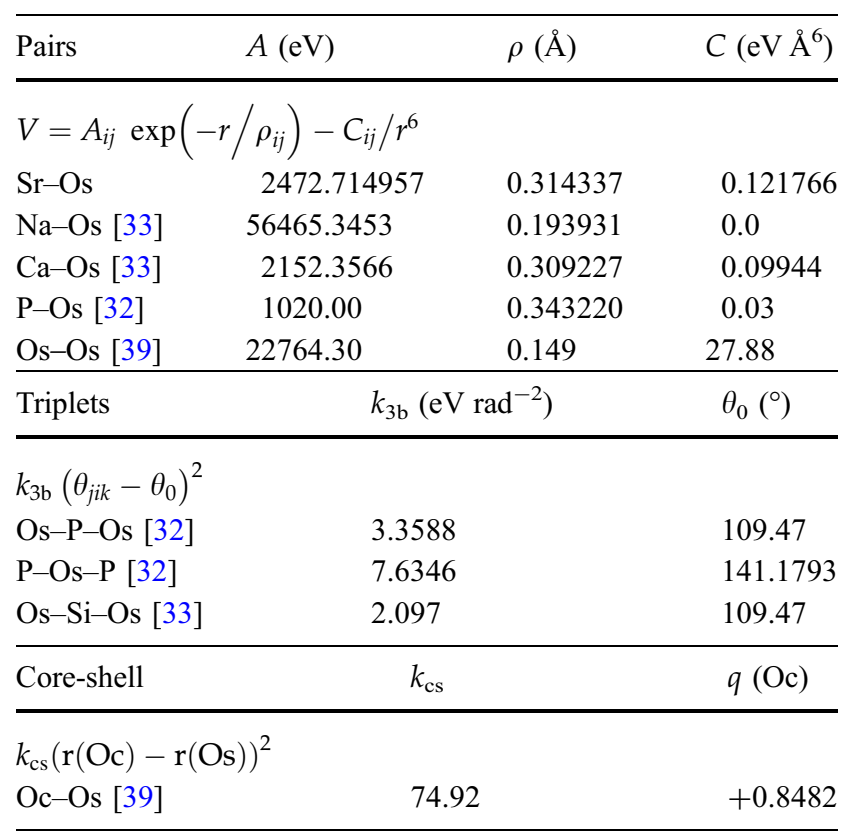

Table 2 The calculated and experimental lattice parameters for strontium crystals using the interatomic potentials developed in this work

\begin{tabular}{lllll}
\hline Material & Parameter & Calc. & Exp. & Diff. \\
\hline $\mathrm{Sr}_{3}\left(\mathrm{PO}_{4}\right)_{2}$ & $a, b$ & $5.491 \AA$ & $5.378 \AA$ & $+2.1 \%$ \\
& $c$ & $19.561 \AA$ & $19.754 \AA$ & $-1.0 \%$ \\
& $\alpha, \beta$ & & $90.0^{\circ}$ & \\
& $\gamma$ & & $120.0^{\circ}$ & \\
$\mathrm{SrO}$ & $a, b, c$ & $5.018 \AA$ & $5.156 \AA$ & $-2.7 \%$ \\
& $\alpha, \beta, \gamma$ & & $90.0^{\circ}$ & \\
$\mathrm{SrSiO}_{3}$ & $a$ & $12.286 \AA$ & $12.333 \AA$ & $-0.4 \%$ \\
& $b$ & $7.147 \AA$ & $7.146 \AA$ & $+0.0 \%$ \\
& $c$ & $10.960 \AA$ & $10.885 \AA$ & $+0.7 \%$ \\
& $\alpha, \gamma$ & & $90.0^{\circ}$ & \\
& $\beta$ & $110.87^{\circ}$ & $111.57^{\circ}$ & $-0.6 \%$ \\
$\mathrm{Sr}_{2} \mathrm{SiO}_{4}$ & $a$ & $9.756 \AA$ & $9.767 \AA$ & $-0.1 \%$ \\
& $b$ & $7.093 \AA$ & $7.084 \AA$ & $+0.1 \%$ \\
& $c$ & $11.061 \AA$ & $11.059 \AA$ & $+0.0 \%$ \\
& $\alpha, \gamma$ & $90.0^{\circ}$ & & \\
& $\beta$ & $148.89^{\circ}$ & $149.24^{\circ}$ & $-0.2 \%$ \\
\hline
\end{tabular}

It is clear that the Sr-Os potential works well in diverse oxide environments, as none of the errors in the lattice parameters or bond angles is larger than $2.7 \%$, and most are considerably smaller. Moreover, the potential performs better, in the sense that the 
errors are smaller, in the silicate and phosphate environments in which it is intended to be used.

\section{Methods}

Our interatomic potential was used in the DLPOLY code [40] to conduct classical MD simulations of a variety of compositions of strontium-containing phosphate glasses based on compositions with $45 \mathrm{~mol} \% \mathrm{P}_{2} \mathrm{O}_{5}, 25 \mathrm{~mol} \% \quad \mathrm{Na}_{2} \mathrm{O}$ and $30 \mathrm{~mol} \% \mathrm{CaO}$, which have been studied both experimentally [17] and in simulation [28]. SrO was substituted both for $\mathrm{CaO}$ (the other alkaline earth oxide) and for $\mathrm{Na}_{2} \mathrm{O}$ to facilitate a comparison to experimental data. The precise compositions and densities studied are given in Table 3. Classical MD was performed on the compositions with 1 and $3 \mathrm{~mol} \% \mathrm{SrO}$, and for each of these compositions, about 3000 atoms were placed independently and randomly into a cubic periodic box to give the appropriate density (Table 3) subject only to the constraint that atoms not be placed closer than $85-90 \%$ of their expected interatomic distances to avoid unphysical starting configurations. Three independent configurations were prepared for each of these compositions. Following an instantaneous relaxation at zero temperature, the classical models were run in an NVT ensemble for 50 ps at $2500 \mathrm{~K}$, before being cooled in a series of runs in NVT ensembles of length $50 \mathrm{ps,} \mathrm{decreasing} \mathrm{the} \mathrm{tempera-}$ ture by $200 \mathrm{~K}$ in each run, at a cooling rate of $4 \mathrm{~K} / \mathrm{ps}$. The model was then run in an NVT ensemble at $300 \mathrm{~K}$ for $200 \mathrm{ps}$, the final two-thirds of which formed the production run. Unless otherwise stated, all data are averaged over snapshots taken from this production run and over the three independent models. The timestep was $0.2 \mathrm{fs}$, and the potentials were truncated at $8 \AA$. The Ewald cut-off was $12 \AA$.
First-principles molecular dynamics, in which the interatomic forces are derived from a quantummechanical representation of the electronic structure, was performed on the compositions with $10 \mathrm{~mol} \%$ SrO (Table 3). Due to the greater computational expense of these simulations, they can only be performed on relatively small models and for shorter timescales. In order to ensure adequate sampling of the strontium environment, therefore, two compositions with this rather high $\mathrm{SrO}$ content were modelled. The CP2K code [41] was used to perform Born-Oppenheimer molecular dynamics on these systems. For each composition, about 150 atoms were placed independently and randomly into a cubic periodic box to give the appropriate density (Table 3) again subject to the constraint that atoms not be placed closer than $85-90 \%$ of their expected interatomic distances. The models were then run in an NVT ensemble at $2500 \mathrm{~K}$ for $20 \mathrm{ps,} \mathrm{before} \mathrm{being}$ cooled in a series of runs in NVT ensembles of length $10 \mathrm{ps,} \mathrm{decreasing} \mathrm{the} \mathrm{temperature} \mathrm{by} \mathrm{about}$ $300 \mathrm{~K}$ in each run, corresponding to a cooling rate of $\sim 30 \mathrm{~K} / \mathrm{ps}$. The models were then run in an NVT ensemble at $300 \mathrm{~K}$ for $\sim 40-50 \mathrm{ps}$, the final twothirds of which formed the production run. Unless otherwise stated, all data are averaged over snapshots taken from this production run. The planewave cut-off was $600 \mathrm{eV}$, and the timestep was 1.0 fs. PBE [42] exchange-correlation functionals in the generalised gradient approximation (GGA) were used.

Although the cooling rates for both classical and first-principles models are much faster than those used experimentally, models prepared using these methodologies have given structures in agreement with experiment for silicate [20, 43-45] and phosphate glasses [28, 46], including for compositions intended for implantation.
Table 3 The glass compositions, densities and numbers of atoms studied in this work

\begin{tabular}{|c|c|c|c|c|c|c|c|c|c|c|}
\hline \multirow[t]{2}{*}{ Name } & \multicolumn{4}{|l|}{ Mol\% } & \multirow{2}{*}{$\begin{array}{l}\text { Density } \\
\left(\mathrm{g} / \mathrm{cm}^{3}\right)\end{array}$} & \multicolumn{5}{|c|}{ Numbers of atoms } \\
\hline & $\mathrm{P}_{2} \mathrm{O}_{5}$ & $\mathrm{Na}_{2} \mathrm{O}$ & $\mathrm{CaO}$ & $\mathrm{SrO}$ & & $\mathrm{O}$ & $\mathrm{P}$ & $\mathrm{Na}$ & $\mathrm{Ca}$ & $\mathrm{Sr}$ \\
\hline P45NSr1 & 45.0 & 24.0 & 30.0 & 1.0 & 2.57 & 1872 & 602 & 320 & 200 & 7 \\
\hline $\mathrm{P} 45 \mathrm{NSr} 3$ & 45.0 & 22.0 & 30.0 & 3.0 & 2.58 & 1879 & 604 & 296 & 201 & 20 \\
\hline P45CSr1 & 45.0 & 25.0 & 29.0 & 1.0 & 2.57 & 1867 & 600 & 334 & 193 & 7 \\
\hline $\mathrm{P} 45 \mathrm{CSr} 3$ & 45.0 & 25.0 & 27.0 & 3.0 & 2.58 & 1867 & 600 & 334 & 180 & 20 \\
\hline P45NSr10 & 45.0 & 15.0 & 30.0 & 10.0 & 2.64 & 93 & 30 & 10 & 10 & 3 \\
\hline $\mathrm{P} 45 \mathrm{CSr} 10$ & 45.0 & 25.0 & 20.0 & 10.0 & 2.64 & 93 & 30 & 16 & 7 & 3 \\
\hline
\end{tabular}




\section{Results}

The relationships between the structure and bioactivity of strontium-free phosphate-based glasses have been discussed elsewhere $[9,16,28]$. Therefore, in this work, we concentrate on characterising the strontium environment.

Table 4 shows the bond lengths and coordination numbers for each composition. The $\mathrm{Sr}-\mathrm{O}$ bond lengths for the classical models are between 2.44 and $2.49 \AA$; those for the first-principles models are slightly longer at $2.55-2.57 \AA$. The generalised gradient approximation (GGA) [42] which we have used is known to underbind certain bonds, which would lead to longer bond lengths, and it seems that this is happening here.

Coordination numbers vary from 7.5 to 7.8 for the classical models and are slightly larger for the firstprinciples models, which is likely due to the longer bond lengths as discussed above. Both the $\mathrm{Sr}-\mathrm{O}$ bond lengths and coordination numbers are larger than the $\mathrm{Na}-\mathrm{O}$ and $\mathrm{Ca}-\mathrm{O}$ bond lengths and coordination numbers, which has profound effects on the bioactivity, as explained later. Figure 1 shows partial paircorrelation functions for the three metal-oxygen pairs in a representative composition, which also shows clearly the larger cut-off needed to define the first coordination shell of strontium.

Experimental estimates of the $\mathrm{Sr}-\mathrm{O}$ bond length and coordination number have not been performed in phosphate glasses, to the best of our knowledge, although the bond lengths we find are in good agreement with those from strontium borosilicate glass $(2.52 \AA)$ [47] and strontium tin silicate glass (2.59-2.64 ̊) [48]. The Sr-O coordination number was between four and five in the borosilicate glass, in contradiction to our results, but it was between seven

Table 4 The $\mathrm{Sr}-\mathrm{O}$ bond lengths and coordination numbers (using a cut-off of $3.5 \AA$ ) for the compositions studied

\begin{tabular}{lll}
\hline Name & Bond length $(\AA)$ & Coordination number \\
\hline P45NSr1 & $(2.45 \pm 0.04)$ & $7.8 \pm 0.5$ \\
P45NSr3 & $(2.44 \pm 0.02)$ & $7.6 \pm 0.1$ \\
P45CSr1 & $(2.49 \pm 0.04)$ & $7.5 \pm 0.5$ \\
P45CSr3 & $(2.46 \pm 0.02)$ & $7.5 \pm 0.2$ \\
P45NSr10 & 2.55 & 8.0 \\
P45CSr10 & 2.57 & 8.7 \\
\hline
\end{tabular}

Errors quoted are SDs from the independent configurations and eight for the tin silicate glass and around seven in other computational studies [13, 14].

Some oxygen atoms are bound to two phosphorus atoms, bridging two $\mathrm{PO}_{4}$ tetrahedra; these are known as bridging oxygen (BO) atoms. Modifier ions prefer to bond to non-bridging oxygen (NBO) atoms, which typically carry a greater charge. When there is more than one modifier atom in a glass composition, they compete for these NBOs, leading to different amounts of bonding to $\mathrm{BO}$ and NBO atoms for different modifiers [49]. In our models, the amounts of NBO bound to each modifier are shown in Table 5 . For all compositions and methodologies, Na has the smallest percentage of NBOs, and Ca the most, with Sr between the two.

The bond-angle distribution around the modifier atoms also reveals something of their local environments. As before, there is little difference between compositions, at least for those simulated with the

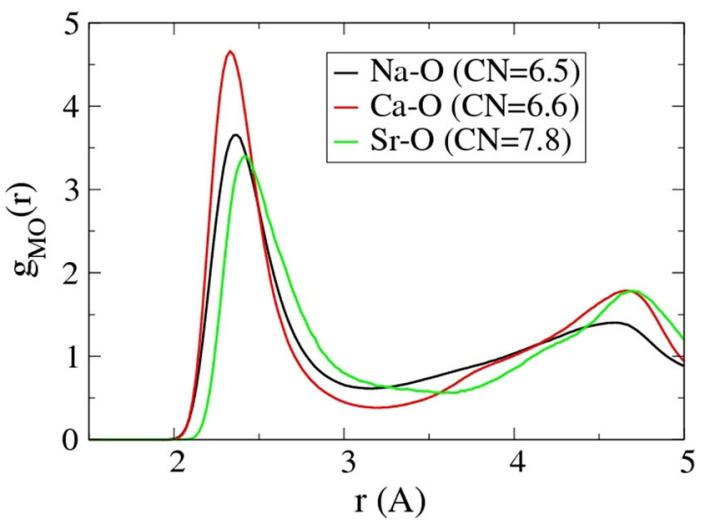

Figure 1 The partial pair-correlation functions $g_{\mathrm{NaO}}(r), g_{\mathrm{CaO}}(r)$ and $g_{\mathrm{SrO}}(r)$ for one model of $\mathrm{P} 45 \mathrm{CSr} 3$, as a representative composition. Coordination numbers are included in the legend; the cut-offs are $3.2 \mathrm{~A}$ for $\mathrm{Na}-\mathrm{O}$ and $\mathrm{Ca}-\mathrm{O}$ and $3.5 \AA$ for $\mathrm{Sr}-\mathrm{O}$.

Table 5 The percentage of non-bridging oxygen atoms in the first coordination shell of the modifier atoms for all compositions

\begin{tabular}{llll}
\hline Name & \multicolumn{2}{l}{ NBO neighbours } \\
\cline { 2 - 4 } & Na (\%) & Sr (\%) & Ca (\%) \\
\hline P45NSr1 & $(80 \pm 1)$ & $(86 \pm 4)$ & $(93 \pm 1)$ \\
P45NSr3 & $(80 \pm 1)$ & $(87 \pm 2)$ & $(93 \pm 1)$ \\
P45CSr1 & $(80 \pm 0)$ & $(91 \pm 1)$ & $(94 \pm 0)$ \\
P45CSr3 & $(80 \pm 0)$ & $(87 \pm 1)$ & $(94 \pm 1)$ \\
P45NSr10 & 86 & 89 & 95 \\
P45CSr10 & 83 & 88 & 92 \\
\hline
\end{tabular}

Errors quoted are SDs from the independent configurations 
same methodology. In Fig. 2, the $\mathrm{O}-\mathrm{M}-\mathrm{O}$ bond-angle distribution, where $\mathrm{M}=\mathrm{Na}, \mathrm{Ca}$ or $\mathrm{Sr}$, is displayed for a representative composition. For each distribution, a very broad distribution of bond angles is seen, reflecting the range of coordination environments and the disorder inherent in the structure. The O-Sr$\mathrm{O}$ bond-angle distribution extends to more acute bond angles than the other two, reflecting strontium's higher coordination number and the need for more acute bond angles to accommodate seven or even eight oxygen atoms in the coordination shell. All three distributions have a broad peak at about $90^{\circ}$, corresponding to a distorted octahedral coordination shell typical of six-coordinated atoms. For O-Sr-O, this peak is at more acute angles because of the greater number of higher-coordinated atoms.

One of the key parameters which controls the bioactivity of glasses implanted into the human body is the network connectivity [21], defined as the average number of bridging oxygen (BO) atoms per phosphorus atom. $\mathrm{CaO} \leftrightarrow \mathrm{SrO}$ substitution is thought unlikely to change the network connectivity or $Q^{n}$ distribution ( $n$ is the number of BOs) because the ratio of $\mathrm{O}$ atoms to $\mathrm{P}$ atoms remains constant. We computed the $Q^{n}$ distribution for the compositions prepared with classical MD to find that they were all almost identical, with $26-27 \% Q^{1}, 68-70 \% Q^{2}$, and $4 \%$ $Q^{3}$ for a network connectivity of 1.77-1.78.

In previous work [28], we and others have identified that one of the most relevant structural features for glasses with $<50 \mathrm{~mol} \% \mathrm{P}_{2} \mathrm{O}_{5}$ (equivalently, phosphate glasses with a network connectivity less than 2 ) is the extent to which the modifier ions bind to different

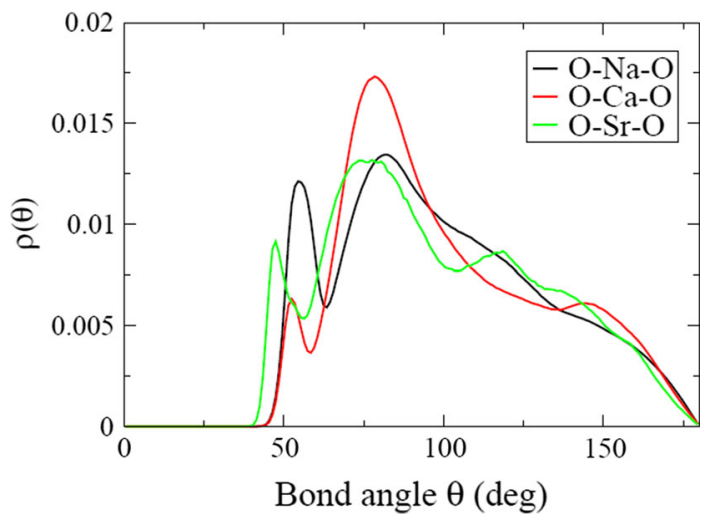

Figure 2 The $\mathrm{O}-\mathrm{Na}-\mathrm{O}$ (black), $\mathrm{O}-\mathrm{Ca}-\mathrm{O}$ (red) and $\mathrm{O}-\mathrm{Sr}-\mathrm{O}$ (green) bond-angle distributions for one model of $\mathrm{P} 45 \mathrm{NSr} 3$, as a representative composition. The cut-offs are $3.2 \AA$ for $\mathrm{Na}-\mathrm{O}$ and $\mathrm{Ca}-\mathrm{O}$ and $3.5 \AA$ for $\mathrm{Sr}-\mathrm{O}$. numbers of phosphate chains. We are able to compute this for the three different modifier ions present in our compositions here, and these results are presented in Table 6. There is a clear difference between the 3.2-3.3 phosphate chains bound to the singly charged sodium ion, and the 3.9 or more chains bound to the doubly charged calcium and strontium ions, as we identified previously for Sr-free glasses [28]. Sr seems to have a similar number-perhaps very slightly larger-of chains bound to it as are bound to Ca.

\section{Discussion}

We have investigated the effects caused by the incorporation of strontium, which has osteogenic and other useful medical properties, into bioactive phosphate glasses, which have a tunable dissolution rate. We have focussed on understanding how strontium changes the atomic structure, and how this is likely to affect the bioactivity.

To do this, we had to develop an accurate potential to represent the interatomic forces involving strontium. The structural features in glasses which most affect the bioactivity are known to occur at the medium-range length scales, i.e., the network connectivity, $Q^{n}$ distribution [21] and phosphate-chain to network-modifier bonding [28]. It is also known that to describe this medium-range structure well using classical molecular dynamics simulations requires a polarizable potential [31]. In order to maximise its utility, therefore, we have developed a polarizable potential that is compatible with previous work in both phosphate and silicate glasses, and we have shown that this potential model represented the crystal structures of strontium oxide, silicates and phosphate, rather well.

Using this new potential, we have shown that the $\mathrm{Sr}-\mathrm{O}$ bond length is $\sim 2.44-2.49 \AA$ and the

Table 6 The average number of fragments bound to each $\mathrm{Na}, \mathrm{Ca}$ or $\mathrm{Sr}$ atom for the glass compositions studied

\begin{tabular}{llll}
\hline \multicolumn{4}{l}{ No. of fragments } \\
\cline { 2 - 4 } & $\mathrm{Na}$ & $\mathrm{Sr}$ & $\mathrm{Ca}$ \\
\hline P45NSr1 & $3.2 \pm 0.1$ & $3.9 \pm 0.2$ & $3.9 \pm 0.1$ \\
P45NSr3 & $3.2 \pm 0.1$ & $4.0 \pm 0.1$ & $3.9 \pm 0.0$ \\
P45CSr1 & $3.2 \pm 0.0$ & $4.1 \pm 0.5$ & $3.9 \pm 0.1$ \\
P45CSr3 & $3.3 \pm 0.0$ & $4.1 \pm 0.2$ & $4.0 \pm 0.1$ \\
\hline
\end{tabular}

Errors quoted are SDs from the independent configurations 
coordination number is 7.5-7.8; both of these quantities are larger than for $\mathrm{Na}-\mathrm{O}$ or $\mathrm{Ca}-\mathrm{O}$, which have typical coordination numbers of less than seven. Although all three modifier ions prefer to bond to non-bridging oxygen atoms, the extent to which each of them does so depends on their field strength [24]. Sodium has the lowest field strength and the lowest percentage of NBOs in its first coordination shell and calcium has the highest field strength and the largest percentage of NBOs in its first coordination shell, while strontium is intermediate between the two. This relationship between field strength and percentage of NBO's takes no account of the different coordination numbers of the three ions. Similarly, the amount of intra-tetrahedral bonding, where two oxygen atoms from one $\mathrm{PO}_{4}$ tetrahedron are bonded to the same modifier atom, varies, being most pronounced in $\mathrm{Na}$, and least visible in $\mathrm{Ca}$, as can be seen by the height of the peak (proportional to the amount of intra-tetrahedral bonding) at about $60^{\circ}$ in the $\mathrm{O}-$ ( $\mathrm{Na}, \mathrm{Ca}, \mathrm{Sr})-\mathrm{O}$ bond-angle distributions.

The network connectivity and $Q^{n}$ distribution are essentially unaffected by the incorporation of strontium. This is to be expected, certainly for the network connectivity, which depends on the ratio of the number of oxygen atoms to the number of phosphorus atoms, which does not change on either $\mathrm{SrO} \leftrightarrow \mathrm{CaO}$ or $\mathrm{SrO} \leftrightarrow \mathrm{Na}_{2} \mathrm{O}$ substitution. The $Q^{n}$ distribution might change, but the amounts of $\mathrm{Sr}$ incorporated here are small, and so the associated change is small.

As for other glasses with similar phosphate contents [28], the dissolution rate of these glasses will be controlled by the different bonding of the modifier ions to the surrounding phosphate chains, which we have characterised. Despite the differences in their local environments in terms of coordination numbers and percentage of non-bridging oxygen atoms in their coordination shell, strontium and calcium bond to a very similar number of fragments. We predict, therefore, that $\mathrm{SrO} \leftrightarrow \mathrm{CaO}$ will not affect the dissolution rate substantially. Substituting $\mathrm{SrO}$ for $\mathrm{Na}_{2} \mathrm{O}$ will affect the dissolution rate, decreasing it with increasing $\mathrm{Sr}$ content, as occurs when $\mathrm{CaO}$ substitutes $\mathrm{Na}_{2} \mathrm{O}$. Based on this prediction, we expect that there will be no structural obstacles to the incorporation of strontium into phosphate-based bioactive glasses for biomedical implantation, and indeed, it may be possible to fine-tune the dissolution rate through careful analysis of the $\mathrm{SrO} / \mathrm{CaO}$ ratio.

\section{Conclusion}

We have conducted first-principles and classical molecular dynamics simulations of various compositions of strontium-containing phosphate glasses, to understand how strontium incorporation will change the glasses' activity when implanted into the body (bioactivity). To perform the classical simulations, we have developed a new interatomic potential, which takes account of the polarizability of the oxygen ions. Our previous work [28-30] on the dissolution of phosphate glasses had shown us that the number of phosphate chains chemically bound to each network modifier is a key parameter to understand the associated effect on the dissolution. We have found that strontium bonds to a similar number of phosphate chains as calcium does. The implication is that $\mathrm{SrO} \leftrightarrow \mathrm{CaO}$ substitution will cause little or no change in the dissolution rate of these glasses and that the bioactivity will remain essentially constant. We conclude, therefore, that strontium could be incorporated into phosphate glass for biomedical applications.

\section{Acknowledgements}

We thank the UK's EPSRC for funding (Grant EP/ J008095/1). Via our membership of the UK's HPC Materials Chemistry Consortium, which is funded by EPSRC (EP/L000202), this work made use of the facilities of HECToR and ARCHER, the UK's national high-performance computing service.

Open Access This article is distributed under the terms of the Creative Commons Attribution 4.0 International License (http://creativecommons.org/ licenses/by/4.0/), which permits unrestricted use, distribution, and reproduction in any medium, provided you give appropriate credit to the original author(s) and the source, provide a link to the Creative Commons license, and indicate if changes were made.

\section{References}

[1] Nielsen SP (2004) The biological role of strontium. Bone 35:583-588

[2] Bonnelye E, Chabadel A, Saltel F, Jurdic P (2008) Dual effect of strontium ranelate: stimulation of osteoblast 
differentiation and inhibition of osteoclast formation and resorption in vitro. Bone 42:129-138

[3] Meunier PJ, Roux C, Seeman E et al (2004) The effects of strontium ranelate on the risk of vertebral fracture in women with postmenopausal osteoporosis. N Engl J Med 350:459-468

[4] Reginster JY, Seeman E, De Vernejoul MC et al (2005) Strontium ranelate reduces the risk of nonvertebral fractures in postmenopausal women with osteoporosis: Treatment of Peripheral Osteoporosis (TROPOS) study. J Clin Endocrinol Metab 90:2816-2822

[5] Marie PJ (2007) Strontium ranelate: new insights into its dual mode of action. Bone 40:S5-S8

[6] Roux C (2007) Antifracture efficacy of strontium ranelate in postmenopausal osteoporosis. Bone 40:S9-S11

[7] Brauer DS, Karpukhina N, Kedia G et al (2013) Bactericidal strontium-releasing injectable bone cements based on bioactive glasses. J R Soc Interface 10:20120647

[8] Porter AT, McEwan AJB, Powe JE et al (1993) Results of a randomised phase III trial to evaluate the efficacy of strontium-89 adjuvant to local field external beam irradiation in the management of endocrine resistant metastatic prostate cancer. Int J Radiat Oncol Biol Phys 25:805-813

[9] Knowles JC (2003) Phosphate based glasses for biomedical applications. J Mater Chem 13:2395-2401

[10] Lakhkar NJ, Lee IH, Kim HW et al (2013) Bone formation controlled by biologically relevant inorganic ions: role and controlled delivery from phosphate-based glasses. Adv Drug Deliv Rev 65:405-420

[11] Fredholm YC, Karpukhina N, Law RV, Hill RG (2010) Strontium containing bioactive glasses: glass structure and physical properties. J Non Cryst Solids 356:2546-2551

[12] Goel A, Rajagopal RR, Ferreira JMF (2011) Influence of strontium on structure, sintering and biodegradation behaviour of $\mathrm{CaO}-\mathrm{MgO}-\mathrm{SrO}-\mathrm{SiO}_{2}-\mathrm{P}_{2} \mathrm{O}_{5}-\mathrm{CaF}_{2}$ glasses. Acta Biomater 7:4071-4080

[13] Xiang Y, Du J (2011) Effect of strontium substitution on the structure of 45S5 bioglasses. Chem Mater 23:2703-2717

[14] Du J, Xiang Y (2012) Effect of strontium substitution on the structure, ionic diffusion and dynamic properties of 45S5 bioactive glasses. J Non Cryst Solids 358:1059-1071

[15] Fujikura K, Karpukhina N, Kasuga T et al (2012) Influence of strontium substitution on structure and crystallisation of bioglass 45S5. J Mater Chem 22:7395-7402

[16] Ahmed I, Lewis M, Olsen I, Knowles JC (2004) Phosphate glasses for tissue engineering: part 1 . Processing and characterisation of a ternary-based $\mathrm{P}_{2} \mathrm{O}_{5}-\mathrm{CaO}-\mathrm{Na}_{2} \mathrm{O}$ glass system. Biomaterials 25:491-499

[17] Abou Neel EA, Chrzanowski W, Pickup DM et al (2009) Structure and properties of strontium-doped phosphate-based glasses. J R Soc Interface 6:435-446
[18] Lakhkar NJ, Abou Neel EA, Salih V, Knowles JC (2009) Strontium oxide doped quaternary glasses: effect on structure, degradation and cytocompatibility. J Mater Sci Mater Med 20:1339-1346

[19] Lakhkar NJ, Abou Neel EA, Salih V, Knowles JC (2011) Titanium and strontium-doped phosphate glasses as vehicles for strontium ion delivery to cells. J Biomater Appl $25: 877-893$

[20] Tilocca A, Cormack AN, de Leeuw NH (2007) The structure of bioactive silicate glasses: new insight from molecular dynamics simulations. Chem Mater 19:95-103

[21] Tilocca A (2009) Review: structural models of bioactive glasses from molecular dynamics simulations. Proc R Soc A 465:1003-1027

[22] Christie JK, Tilocca A (2010) Aluminosilicate glasses as yttrium vectors for in situ radiotherapy: understanding composition-durability effects through molecular dynamics simulations. Chem Mater 22:3725-3734

[23] Christie JK, Tilocca A (2010) Short-range structure of yttrium aluminosilicate glass for cancer radiotherapy: CarParrinello molecular dynamics simulations. Adv Eng Mater 12:B326-B330

[24] Christie JK, Malik J, Tilocca A (2011) Bioactive glasses as potential radioisotope vectors for in situ cancer therapy: investigating the structural effects of yttrium. Phys Chem Chem Phys 13:17749-17755

[25] Christie JK, Pedone A, Menziani MC, Tilocca A (2011) Fluorine environment in bioactive glasses: $a b$ initio molecular dynamics simulations. J Phys Chem B 115:2038-2045

[26] Christie JK, Tilocca A (2012) Integrating biological activity into radioisotope vectors: molecular dynamics models of yttrium-doped bioactive glasses. J Mater Chem 22:1202312031

[27] Christie JK, Tilocca A (2012) Molecular dynamics simulations and structural descriptors of radioisotope glass vectors for in-situ radiotherapy. J Phys Chem B 116:12614-12620

[28] Christie JK, Ainsworth RI, Di Tommaso D, de Leeuw NH (2013) Nanoscale chains control the solubility of phosphate glasses for biomedical applications. J Phys Chem B 117:10652-10657

[29] Christie JK, Ainsworth RI, de Leeuw NH (2014) Ab initio molecular dynamics simulations of structural changes associated with the incorporation of fluorine in bioactive phosphate glasses. Biomaterials 35:6164-6171

[30] Ainsworth RI, Christie JK, de Leeuw NH (2014) On the structure of biomedical silver-doped phosphate-based glasses from molecular dynamics simulations. Phys Chem Chem Phys 16:21135-21143

[31] Tilocca A (2008) Short- and medium-range structure of multicomponent bioactive glasses and melts: an assessment 
of the performances of shell-model and rigid-ion potentials. J Chem Phys 129:084504

[32] Ainsworth RI, Di Tommaso D, Christie JK, de Leeuw NH (2012) Polarizable force field development and molecular dynamics study of phosphate-based glasses. J Chem Phys 137:234502

[33] Tilocca A, de Leeuw NH, Cormack AN (2006) Shell-model molecular dynamics calculations of modified silicate glasses. Phys Rev B 73:104209

[34] Gale JD, Rohl AL (2003) The general utility lattice program. Mol Simul 29:291-341

[35] Leshchenko PP, Shevchenko AV, Lykova LN et al (1982) Inorg Mater 18:1013-1016

[36] Nishi F (1997) Strontium metasilicate, $\mathrm{SrSiO}_{3}$. Acta Cryst C53:534-536

[37] Catti M, Gazzoni G, Ivaldi G (1983) Structures of twinned $\beta-\mathrm{Sr}_{2} \mathrm{SiO}_{4}$ and of $\alpha^{\prime}-\mathrm{Sr}_{1.9} \mathrm{Ba}_{0.1} \mathrm{SiO}_{4}$. Acta Cryst C39:29-34

[38] Zachariasen WH (1948) The crystal structure of the normal orthophosphates of barium and strontium. Acta Cryst 1:263-265

[39] Sanders MJ, Leslie M, Catlow CRA (1984) Interatomic potentials for $\mathrm{SiO}_{2}$. J Chem Soc Chem Commun 19:1271-1273

[40] Todorov IT, Smith W, Trachenko K, Dove MT (2006) DL_POLY_3: new dimensions in molecular dynamics simulations via massive parallelism. J Mater Chem 16:1911-1918

[41] Hutter J, Iannuzzi M, Schiffmann F, VandeVondele J (2014) CP2K: atomistic simulations of condensed matter systems. WIREs Comput Mol Sci 4:15-25
[42] Perdew JP, Burke K, Ernzerhof M (1996) Generalized gradient approximation made simple. Phys Rev Lett 77:3865-3868

[43] Vollmayr K, Kob W, Binder K (1996) Cooling-rate effects in amorphous silica: a computer-simulation study. Phys Rev B 54:15808-15827

[44] Jund P, Jullien R (1999) Computer investigation of the energy landscape of amorphous silica. Phys Rev Lett 83:2210-2213

[45] Tilocca A, de Leeuw NH (2006) Structural and electronic properties of modified sodium and soda-lime silicate glasses by Car-Parrinello molecular dynamics. J Mater Chem 16:1950-1955

[46] Du J, Kokou L, Rygel JL, Chen Y, Pantano CG, Woodman R, Belcher J (2011) Structure of cerium phosphate glasses: molecular dynamics simulations. J Am Ceram Soc 94:2393-2401

[47] McKeown DA, Kot WK, Pegg IL (2003) X-ray absorption studies of the local strontium environments in borosilicate waste glasses. J Non-Cryst Sol 317:290-300

[48] Johnson JA, Urquidi A, Holland D, Johnson CE, Appleyard PG (2007) Strontium environment transition in tin silicate glasses by neutron and X-ray diffraction. J Non-Cryst Sol 353:4084-4092

[49] Varshneya AK (1993) Fundamentals of inorganic glasses. Academic Press 